\title{
Optimization of pure platelet-rich plasma preparation: A comparative study of pure platelet-rich plasma obtained using different centrifugal conditions in a single-donor model
}

\author{
WENJING YIN*, HAITAO XU*, JIAGEN SHENG, ZHENZHONG ZHU, \\ DONGXU JIN, PEICHUN HSU, XUETAO XIE and CHANGQING ZHANG \\ Department of Orthopaedic Surgery, Shanghai Jiao Tong University \\ Affiliated Sixth People's Hospital, Shanghai 200233, P.R. China
}

Received March 7, 2016; Accepted February 24, 2017

DOI: $10.3892 /$ etm.2017.4726

\begin{abstract}
While it has been proved that centrifugal conditions for pure platelet-rich plasma (P-PRP) preparation influence the cellular composition of P-PRP obtained, the optimal centrifugal conditions to prepare P-PRP have not yet been identified. In the present study, platelet-containing plasma (PCP) was prepared with the first-spin of different double-spin methods and P-PRP was prepared with different double-spin methods. Whole-blood analysis was performed to evaluate the cellular composition of PCP and P-PRP. The basal and ADP-induced CD62P expression rates of platelets were assessed by flow cytometry to evaluate the function of platelets in PCP and P-PRP. Enzyme-linked immune sorbent assay was performed to quantify interleukin-1 $\beta$, tumor necrosis factor- $\alpha$, platelet-derived growth factor $\mathrm{AB}$ and transforming growth factor $\beta 1$ concentrations of PCP and P-PRP. Correlations between the cellular characteristics and cytokine concentrations of P-PRP were analyzed by Pearson correlation analysis. Effects of P-PRP on the proliferation, survival and migration of human bone marrow-derived mesenchymal stem cells and human articular chondrocytes were evaluated by a Cell Counting Kit- 8 assay, live/dead staining and Transwell assay, respectively. The results showed that centrifugation at $160 \mathrm{xg}$ for $10 \mathrm{~min}$ and $250 \mathrm{x}$ g for $15 \mathrm{~min}$ successively captured and concentrated platelets and growth factors significantly more efficiently with preservation of platelet function compared with
\end{abstract}

Correspondence to: Professor Changqing Zhang or Dr Xuetao Xie, Department of Orthopaedic Surgery, Shanghai Jiao Tong University Affiliated Sixth People's Hospital, 600 Yishan Road, Shanghai 200233, P.R. China

E-mail: zhangcq@sjtu.edu.cn

E-mail: xuetaoxie@163.com

${ }^{*}$ Contributed equally

Key words: platelet-rich plasma, cytokines, leukocyte reduction procedures, cell proliferation, cell migration assays, cell survival other conditions $(\mathrm{P}<0.05)$. The correlation analysis showed that the similar leukocyte concentrations and leukocyte-reducing efficiencies resulted in similar pro-inflammatory cytokine concentrations in P-PRP $(\mathrm{P}>0.05)$ and the maximization of platelet concentration, platelet enrichment factor, platelet capture efficiency and platelet function resulted in the maximization of growth factor concentrations in P-PRP obtained using the optimal conditions $(\mathrm{P}<0.05)$. Compared with P-PRP obtained under other conditions, P-PRP obtained under the optimal conditions significantly promoted the proliferation and migration of cells $(\mathrm{P}<0.05)$ and did not alter cell survival ( $P>0.05)$. Therefore, centrifugation at $160 \mathrm{x}$ g for $10 \mathrm{~min}$ and $250 \mathrm{x} g$ for 15 min successively with removal of the buffy coat as a crucial step may provide an optimal preparation system of P-PRP for clinical application.

\section{Introduction}

Platelet-rich plasma (PRP), an autologous derivative of whole blood that contains concentrated platelets, has been advocated as a way to introduce increased concentrations of growth factors that are known to have beneficial effects on tissue regeneration, including platelet-derived growth factor (PDGF), transforming growth factor (TGF), insulin-like growth factor (IGF) and vascular endothelial growth factor (VEGF) (1), to injured tissue in an attempt to aid in tissue regeneration (2-5). However, leukocytes in PRP release pro-inflammatory cytokines, such as interleukin-1 $\beta$ (IL-1 $\beta$ ) and tumor necrosis factor- $\alpha(\mathrm{TNF}-\alpha)$ to counteract the beneficial effects of growth factors on tissue regeneration (6-8). Consequently, effort has been put into the depletion of leukocytes from PRP to prepare pure PRP (P-PRP) over the past few years.

Multiple systems have been developed to offer an easy, cost-effective strategy to prepare P-PRP, including selective blood filtration methods $(7,9)$ and centrifugation methods $(3,10,11)$. The latter are widely used due to their feasibility and comparatively lower cost (12). The basic principle of preparing P-PRP with centrifugation methods is that it allows platelets to settle and to concentrate in the lower layer of the plasma, and therefore to become separated from the upper layer. In addition, erythrocytes and leukocytes settle faster than 
platelets, allowing for individual separation from plasma (13). This phenomenon is governed by Stokes' law (14), which states that the sedimentation rates of particles in a liquid environment are positively correlated to the mass of particles and the sedimentation force the particles are exposed to. Regarding P-PRP preparation, a greater centrifugal force enhances the sedimentation force and hence the difference of the sedimentation rates between erythrocytes, leukocytes and platelets, and a longer centrifugal duration enhances the sedimentation duration and hence guarantees the capture and enrichment of platelets due to the difference in the sedimentation rate. A great centrifugal force and long centrifugal duration separates platelets from the plasma to form a 'buffy coat' together with leukocytes and hence, they are unable to be separated from leukocytes. Thus, the currently used centrifugal conditions to prepare P-PRP require to be optimized $(10,15)$. Although the cellular characteristics of PRP obtained using different preparation systems have been evaluated by numerous studies in order to identify the optimal centrifugal conditions for the preparation of leukocyte-rich PRP and P-PRP, the cellular characterization of PRP is typically limited to PRP obtained using currently available preparation systems that have been developed to date, while the optimal centrifugal conditions may have remained to be determined (10).

The first purpose of the present study was to identify the optimal centrifugal conditions to capture and concentrate platelets while depleting erythrocytes and leukocytes. As the optimal centrifugal conditions for platelet capture and enrichment may be different $(10,15)$, double-spin methods were applied to capture platelets with the first spin and concentrate them further with the second spin. As cytokines released from platelets and leukocytes are thought to be the effective components of PRP, the second purpose of the study was to evaluate cytokine concentrations in P-PRP obtained using different conditions and the correlations between cytokine concentrations and the cellular characteristics of P-PRP. The third purpose of the study was to evaluate the in vitro effects of P-PRP obtained using different conditions on cells.

\section{Materials and methods}

Subjects. The study was performed in accordance with the principles of the Declaration of Helsinki. The Independent Ethics Committee of the Sixth People's Hospital Affiliated to Shanghai Jiao Tong University (Shanghai, China) approved the study protocols for collecting samples and their use for scientific experiments.

A total of 80 healthy volunteers (46 men and 34 women; age, 21-60 years) were included in the study for blood donation. Healthy adults who agreed to participate in the study and gave informed consent were included. The exclusion criterion was a medical history of relevant diseases or consumption of any medications known to affect platelet function or concentration for 21 days prior to blood collection.

Volunteers were randomly divided into two groups $(n=40)$. The blood collected from volunteers of the single-spin group was used to identify the optimal centrifugal conditions for the first spin and the blood collected from volunteers of the double-spin group was used to identify the optimal centrifugal conditions for the second spin.
Blood collection. Approximately $216 \mathrm{ml}$ venous blood collected by a licensed phlebotomist using a 19-gauge blood collection needle was added into $24 \mathrm{ml}$ of acid-citrate dextrose solution A anti-coagulant to prepare $240 \mathrm{ml}$ of anti-coagulated whole blood for each volunteer. A single-donor model was applied to minimize potential confounding variables (1). The anti-coagulated whole blood was split into six aliquots of $40 \mathrm{ml}$ in 50-ml centrifuge tubes (Corning, Lowell, MA, USA) and subjected to the first spin within $30 \mathrm{~min}$ after collecting in an automated tabletop centrifuge (Ankel TDL-5-A; Anting Scientific Instrument Factory, Shanghai, China). A blood collection tube coated with K2 EDTA (BD Vacutainer; BD Biosciences, Franklin Lakes, NJ, USA) was used to collect $2 \mathrm{ml}$ of venous blood for whole-blood analysis.

Centrifugal conditions for the first spin. In existing systems, a centrifugal duration of 10-15 min is most frequently applied and short enough to be acceptable in clinical practice; therefore, it was selected as the centrifugal duration for the first and the second spin. Based on the results of a previous study, centrifugal conditions of $<110 \mathrm{x}$ g for $15 \mathrm{~min}$ cannot separate erythrocytes and leukocytes from concentrated platelets, while a centrifugal force $>180 \mathrm{x}$ g for $10 \mathrm{~min}$ separates platelets from plasma to form a 'buffy coat' together with leukocytes and hence they are unable to be separated from leukocytes (unpublished data). Thus, in the present study, the first spin was performed at $110 \mathrm{x} g$ for $15 \mathrm{~min}(110 \times 15), 130 \times 10,130 \times 15$, $160 \times 10,160 \times 15$ or $180 \times 10$ at room temperature.

After the first spin, the blood was separated into three components: Erythrocytes at the bottom, buffy coat in the middle and platelet-containing plasma (PCP) at the top. Although the buffy coat contains concentrated platelets, it also contains concentrated leukocytes. Thus, the bottom and middle layers were discarded to deplete leukocytes and erythrocytes, and the PCP was transferred to a new tube and subjected to the second spin to prepare P-PRP in the second-spin group, which was collected and measured for volume by gentle aspiration with a 5-ml graduated pipette (Jet Biofil, Guangzhou, China). All procedures were performed by the same operator (WY).

Centrifugal conditions for the second spin. The first spin was performed under the optimal conditions according to the results of the test for conditions for the first spin. After the first spin, PCP was collected by gentle aspiration and transferred to a new 50-ml centrifuge tube. Care was taken to avoid contamination of buffy coat and erythrocytes. Based on the results of a previous study (10), the optimal centrifugal conditions to concentrate platelets may be $250 \times 15$ and therefore, the second spin was performed at $180 \times 10,180 \times 15,250 \times 10,250 \times 15$, $450 \times 10$ or $450 \times 15$ at room temperature. After the second spin, the supernatant platelet-poor plasma was discarded by gentle aspiration. Subsequently, the pellets containing platelets were resuspended in the residual supernatant to obtain a total of $4 \mathrm{ml}$ of P-PRP. All procedures were performed by the same operator (HX).

Whole-blood analysis. Whole-blood analysis was performed using an automatic hematology analyzer (XS-800i; Sysmex, Kobe, Japan) in the clinical laboratory of the hospital to determine the concentration of erythrocytes, leukocytes and 
platelets in the whole blood,PCP obtained by different first-spin conditions and P-PRP obtained by different second-spin conditions. Platelet capture efficiencies, platelet enrichment factors, leukocyte-reducing efficiencies and erythrocyte-reducing efficiencies of PCP and P-PRP were calculated according to formulas given in Fig. 1.

Analysis of platelet activation status. Platelet activation statuses of PCP obtained from different first-spin conditions and P-PRP obtained from different second-spin conditions under basal conditions and after incubation with $200 \mu \mathrm{M}$ adenosine diphosphate (ADP; Sigma-Aldrich; Merck KGaA, Darmstadt, Germany) were analyzed to evaluate platelet function (10). In brief, PCP and P-PRP under basal condition or after incubation with $200 \mu \mathrm{M}$ ADP for $5 \mathrm{~min}$ at room temperature were incubated with fluorescein isothiocyanate (FITC)-labeled anti-CD62P (BD Pharmingen, Oxford, UK) or FITC-labeled control antibody (BD Pharmingen) for $30 \mathrm{~min}$ at room temperature. The platelet activation statuses of PCP and P-PRP under basal conditions and after exogenous activation were determined by assessing the CD62P expression rates of platelets by flow cytometry and guavaSoft (Guava easyCyte 8HT flow cytometry system; Millipore, Billerica, MA, USA).

Quantification of cytokine concentrations in PCP and $P$-PRP. PCP obtained from different first-spin conditions and P-PRP obtained using different second-spin conditions were activated with $10 \% \mathrm{CaCl}_{2}$ (final concentration, $22.8 \mathrm{mM}$ ). Subsequently, the formulations were incubated at $37^{\circ} \mathrm{C}$ in a humidified atmosphere with $5 \% \mathrm{CO}_{2}$ for $2 \mathrm{~h}$. At the end of the incubation period, the formulations were centrifuged at $2,800 \times 15$ and the supernatant was collected and stored at $-80^{\circ} \mathrm{C}$ until analysis. The supernatant was assayed for IL-1 $\beta$, tumor necrosis factor (TNF)- $\alpha$, platelet-derived growth factor (PDGF)-AB and transforming growth factor (TGF)- $\beta 1$ with Quantikine Human Immunoassay kits (R\&D Systems, Minneapolis, MN, USA) according to the manufacturer's protocol.

Isolation and culture of cells. Human bone marrow-derived mesenchymal stem cells (hBMSCs) were isolated as described elsewhere (16). In brief, bone marrow aspirates were harvested from the greater trochanter during femur fracture surgery, anti-coagulated with preservative-free heparin $(1,000 \mathrm{U} / \mathrm{ml})$, filtered with a $70-\mathrm{mm}$ filter mesh and suspended in $\alpha$-modification of minimum essential medium (Sigma-Aldrich; Merck KGaA) containing 10\% fetal bovine serum (FBS; Gibco; Thermo Fisher Scientific, Inc., Waltham, MA, USA) and $1 \%$ antibiotics (penicillin $\mathrm{G}$ and streptomycin; Gibco; Thermo Fisher Scientific, Inc.) at $37^{\circ} \mathrm{C}$ in a humidified atmosphere containing $5 \% \mathrm{CO}_{2}$. The medium was changed after $48 \mathrm{~h}$ to remove non-adherent cells and thereafter every three days. Cells of the third passage were used for this study.

Human articular chondrocytes (hACs) were isolated according to the protocol of a previous study (17). In brief, articular cartilage samples were obtained from patients undergoing total knee replacement surgery, minced into small pieces and digested with collagenase II (Sigma-Aldrich; Merck KGaA). The released hACs were then cultured in 6-well plates at a density of $2.5 \times 10^{5}$ cells/well in Dulbecco's modified Eagle's medium/Ham's F-12 50/50 mix (Corning, Inc., Corning, NY, USA) containing $10 \%$ FBS and $1 \%$ antibiotics at $37^{\circ} \mathrm{C}$ in a humidified atmosphere with $5 \% \mathrm{CO}_{2}$. The medium was changed after $48 \mathrm{~h}$ to remove non-adherent cells and every three days thereafter. Cells of the third passage were used for this study.

Cell proliferation assay. Cells were seeded in 96-well plates at a density of 4,000 cells/well and cultured for $24 \mathrm{~h}$ in FBS-free medium. Cells were then cultured in medium supplemented with $10 \%$ (volume/volume) of P-PRP obtained from different conditions for seven days. The proliferation of cells grown in the presence of P-PRP formulations was evaluated on days 1, 4 and 7 using a Cell Counting Kit-8 (CCK-8, Dojindo, Kumamoto, Japan) according to the manufacturer's instructions. In brief, $10 \mu \mathrm{l}$ CCK-8 solution was added to each well containing $100 \mu \mathrm{l}$ medium and incubated for $3 \mathrm{~h}$. The absorbance value was measured with a microplate reader (Bio-Rad Laboratories, Inc., Hercules, CA, USA) at $450 \mathrm{~nm}$.

Cell survival analysis. Cells were seeded in 6-well plates at a density of $1 \times 10^{5}$ cells/well, serum-starved for $24 \mathrm{~h}$ and cultured in medium supplemented with $10 \%$ P-PRP obtained from different conditions for seven days. Cells were then subjected to live/dead staining using a Cell Viability Imaging kit (Thermo Fisher Scientific, Inc.) according to the manufacturer's instructions. The survival of cells was observed using an inverted microscope (Leica, Wetzlar, Germany) and counted in five randomly selected fields per well.

Cell migration analysis. Effects of P-PRP formulations on cell migration were evaluated using a Transwell assay as described previously (18). In brief, confluent cells were serum-starved for $24 \mathrm{~h}$, detached by $0.25 \%$ trypsin-EDTA (Invitrogen; Thermo Fisher Scientific, Inc.) and seeded at a density of $1 \times 10^{5} /$ well in the upper chambers of 24-well Transwell systems (Corning, Inc.). Medium containing 10\% P-PRP obtained from different conditions was then added into the lower chambers. After $24 \mathrm{~h}$ of incubation, the cells on the upper surface of the membranes were removed with a cotton swab and the cells migrated to the lower surface were fixed with $4 \%$ paraformaldehyde, stained using $0.5 \%$ crystal violet for $10 \mathrm{~min}$, observed using an microscope and counted on five randomly selected fields per membrane.

Statistical analysis. Data were analyzed using the Statistical Package for Social Sciences version 22.0 (IBM SPSS, Armonk, NY, USA). Values are expressed as the mean \pm standard deviation or number of volunteers as appropriate. The independent-samples Student's t-test was performed to analyze differences in continuous data between the single- or double-spin groups and the chi-square test was used to analyze the difference between groups regarding gender. One-way analysis of variance and Bonferroni's post-hoc test were performed to analyze the difference in continuous data among conditions. Pearson correlation analysis was conducted to analyze linear correlations between variables. $\mathrm{P}<0.05$ was considered to indicate a statistically significant difference. 
Platelet capture efficiency $(\%)=$

Volume of PCP or P-PRP $(\mathrm{ml}) \times$ platelet concentration of PCP or P-PRP $\left(10^{\%} / \mathrm{l}\right)$

Volume of the whole blood $(\mathrm{ml}) \times$ platelet concentration of the whole blood $\left(10^{9} / \mathrm{l}\right)$

Platelet enrichment factor $($ fold $)=$

Platelet concentration of PCP or P-PRP $\left(10^{\%} / 1\right)$

Platelet concentration of the whole blood $\left(10^{\%} / 1\right)$

Leukocyte-reducing efficiency $(\%)=$

Volume of PCP or P-PRP $(\mathrm{ml}) \times$ leukocyte concentration of PCP or P-PRP $\left(10^{9} / \mathrm{l}\right)$

$100 \%-$ Volume of the whole blood $(\mathrm{ml}) \times$ leukocyte concentration of the whole blood $\left(10^{9} / \mathrm{l}\right)$

Erythrocyte-reducing efficiency $(\%)=$

Volume of PCP or P-PRP $(\mathrm{ml}) \times$ erythrocyte concentration of PCP or P-PRP $\left(10^{12} / \mathrm{l}\right)$

$100 \%$

Volume of the whole blood $(\mathrm{ml}) \times$ erythrocyte concentration of the whole blood $\left(10^{12} / \mathrm{l}\right)$

Figure 1. Formulas for the calculation of the characteristics of PCP and P-PRP. PCP, platelet-containing plasma; P-PRP, pure platelet-rich plasma.

\section{Results}

General information of subjects and blood cell concentrations. General information and blood cell concentrations of volunteers are listed in Table I. No statistically significant differences were found between the single-spin group and the double-spin group regarding all variables.

Characteristics of PCP obtained from different first-spin conditions. The volume of PCP obtained from 160x10 was significantly higher than that obtained from 110x15 $(\mathrm{P}<0.001)$ and comparable with that obtained by $130 \times 10(\mathrm{P}=0.096)$, $130 \times 15(\mathrm{P}>0.999), 160 \times 15(\mathrm{P}>0.999)$ and $180 \times 10(\mathrm{P}=0.189$; Fig. 2A).

The platelet concentration of PCP obtained using 160x 10 was significantly higher than that of PCP obtained using 110x15 ( $\mathrm{P}<0.001), 130 \times 10(\mathrm{P}<0.001), 160 \times 15(\mathrm{P}<0.001)$ and $180 \times 10(\mathrm{P}<0.001)$, and comparable with that obtained using 130x15 (P>0.999; Fig. 2B). Similarly, the platelet enrichment factor of PCP obtained using 160x10 was significantly higher than that of PCP obtained from $110 \times 15(\mathrm{P}<0.001), 130 \times 10$ $(\mathrm{P}<0.001), 160 \times 15(\mathrm{P}<0.001)$ and $180 \times 10(\mathrm{P}<0.001)$, and was not significantly different from that obtained from $130 \times 15$ (P>0.999; Fig. 2C).

The platelet capture efficiency of PCP obtained using $160 \times 10$ was significantly higher than that obtained from $110 \times 15(\mathrm{P}<0.001), 130 \times 10(\mathrm{P}<0.001), 130 \times 15(\mathrm{P}<0.001)$, 160x15 ( $\mathrm{P}<0.001)$ and 180x10 $(\mathrm{P}<0.001$; Fig. 2D).

PCP obtained using different conditions had comparable leukocyte concentrations $(\mathrm{P}=0.214$; Fig. $2 \mathrm{E})$, erythrocyte concentrations $(\mathrm{P}=0.146$; Fig. $2 \mathrm{~F})$, leukocyte-reducing efficiencies $(\mathrm{P}=0.400$; Fig. $2 \mathrm{G})$, erythrocyte-reducing efficiencies $(\mathrm{P}=0.634$; Fig. $2 \mathrm{H})$, basal $\mathrm{CD} 62 \mathrm{P}$ expression rates of platelets ( $\mathrm{P}=0.451$; Fig. 2I) and ADP-induced $\mathrm{CD} 62 \mathrm{P}$ expression rates of platelets $(\mathrm{P}=0.304$; Fig. $2 \mathrm{~J})$.

Similar to the results on the platelet capture efficiency, $\mathrm{PCP}$ obtained using 160x10 had the highest PDGF-AB concentration compared to that obtained using $110 \times 15(\mathrm{P}<0.001), 130 \times 10$ $(\mathrm{P}<0.001), 130 \times 15(\mathrm{P}=0.027), 160 \times 15(\mathrm{P}<0.001)$ and $180 \times 10$ $(\mathrm{P}<0.001$; Fig. 3A). In addition, PCP obtained using $160 \times 10$ had the highest TGF- $\beta 1$ concentration compared with that in PCP obtained using 110x15 $(\mathrm{P}<0.001), 130 \times 10(\mathrm{P}<0.001)$, $130 \times 15(\mathrm{P}=0.037), 160 \times 15(\mathrm{P}<0.001)$ and $180 \times 10(\mathrm{P}<0.001$; Fig. 3B).

Similar to the results on the erythrocyte- and leukocyte-associated characteristics of PCP, the differences in IL-1 $\beta$ and TNF- $\alpha$ concentrations among conditions were not significant ( $\mathrm{P}=0.467$ and $\mathrm{P}=0.461$, respectively; Fig. $3 \mathrm{C}$ and $\mathrm{D})$.

The aforementioned results demonstrated that, compared with other conditions, centrifugation at 160x10 had the highest platelet capture efficiency and achieved the highest growth factor concentrations, while the concentration and reducing efficiency of leukocytes and erythrocytes, pro-inflammatory cytokine concentrations and platelet activation status were similar to those obtained by other conditions. In addition, the platelet concentration and platelet enrichment factor of PCP obtained by $160 \times 10$ were higher than those obtained using other conditions except 130x15. Therefore, 160x10 was designated as the optimal centrifugation conditions for the first spin.

Characteristics of $P$-PRP obtained using different second-spin conditions. As shown in Fig. 4A, the volumes of P-PRP obtained from different conditions were constant $(\mathrm{P}=0.563$; Fig. 4A). One-way analysis of variance indicated that the difference in platelet concentration of P-PRP among the conditions was significant $(\mathrm{P}=0.003)$. However, the Bonferroni post-hoc test was unable to identify any significant difference between conditions ( $\mathrm{P}>0.05$; Fig. 4B). The platelet enrichment factor of P-PRP obtained using 250x15 was significantly higher than that obtained using $180 \times 10(\mathrm{P}<0.001), 180 \times 15(\mathrm{P}<0.001)$ and $250 \times 10(\mathrm{P}<0.001)$. However, the platelet enrichment factor did not further increase by preparation using 450x10 ( $\mathrm{P}>0.999)$ and 450x15 (P>0.999) compared with 250x15 (Fig. 4C). 
Table I. General information and blood cell concentrations in blood from grouped volunteers.

\begin{tabular}{lccr}
\hline Parameter & Single-spin group & Double-spin group & P-value \\
\hline Number of volunteers & 40 & 40 & \\
Gender (male/female) & $24: 16$ & $22: 18$ & 0.653 \\
Age (years) & $40.78 \pm 10.18$ & $42.18 \pm 10.57$ & 0.548 \\
Leukocyte concentration $\left(\times 10^{9} / 1\right)$ & $6.24 \pm 1.51$ & $6.06 \pm 1.50$ & 0.595 \\
Erythrocyte concentration $\left(\times 10^{12} / 1\right)$ & $4.88 \pm 0.51$ & $4.90 \pm 0.48$ & 0.867 \\
Platelet concentration $\left(\times 10^{9} / 1\right)$ & $238.30 \pm 36.29$ & $234.25 \pm 37.53$ & 0.625 \\
\hline
\end{tabular}

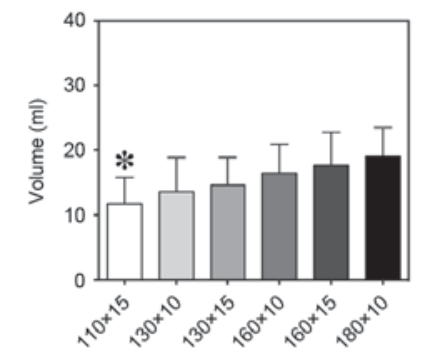

E
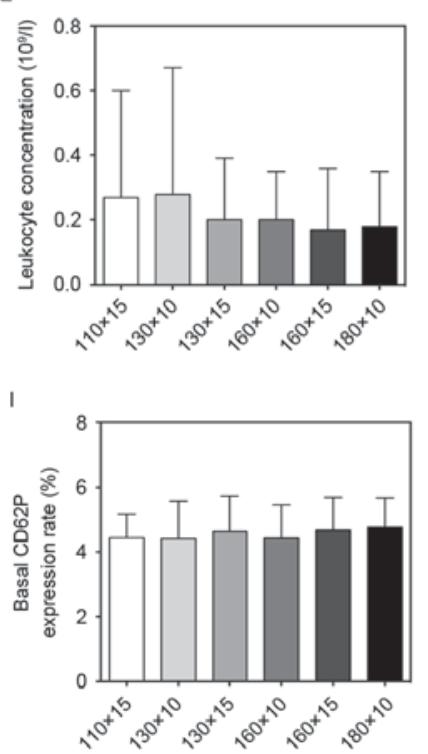

B

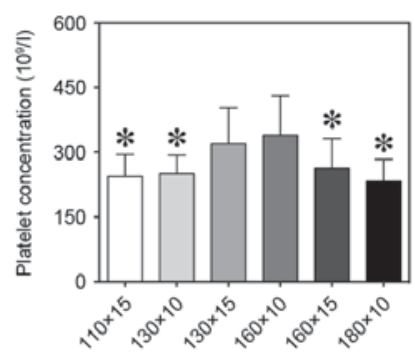

F
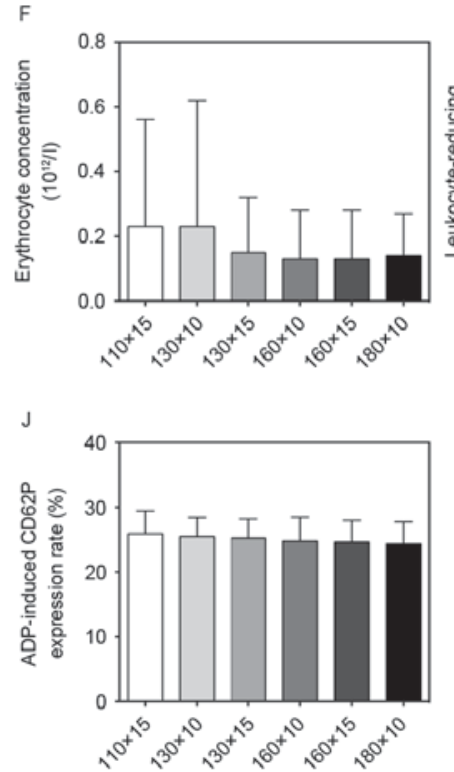

C

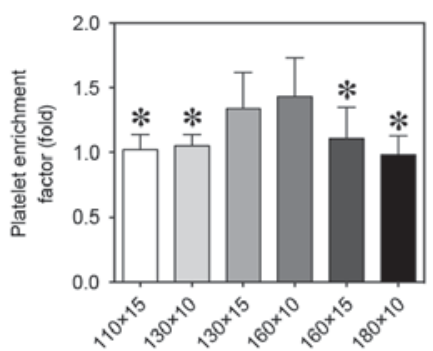

G
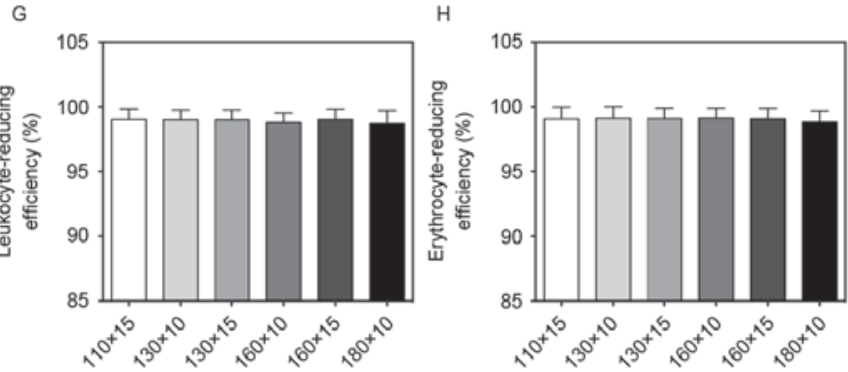

Figure 2. Cellular characteristics of platelet-containing plasma obtained using different conditions. (A) Volume, (B) platelet concentration, (C) platelet enrichment factor, (D) platelet capture efficiency, (E) leukocyte concentration, (F) erythrocyte concentration, (G) leukocyte-reducing efficiency, (H) erythrocyte-reducing efficiency, (I) basal CD62P expression rate and ( $\mathrm{J}$ ) ADP-induced CD62P expression rate. Values are expressed as the mean \pm standard deviation. * $\mathrm{P}<0.05$ compared with $160 \times 10$. ADP, adenosine diphosphate; 160x10, centrifugation at $160 \times \mathrm{g}$ for $10 \mathrm{~min}$.

The platelet capture efficiency of P-PRP obtained by centrifugation at $250 \mathrm{x} 15$ was significantly higher than that obtained at 180x10 $(\mathrm{P}<0.001), 180 \times 15(\mathrm{P}<0.001)$ and 250x10 $(P<0.001)$. Similar to the results on the platelet enrichment factor of P-PRP, the platelet capture efficiency did not further increase by preparation using 450x10 $(\mathrm{P}>0.999)$ and $450 \times 15$ (P>0.999; Fig. 4D).

Similar to the results of the first spin, P-PRP obtained using different conditions provided comparable leukocyte concentrations ( $\mathrm{P}=0.839$; Fig. 4E), erythrocyte concentrations $(\mathrm{P}=0.997$; Fig. 4F), leukocyte-reducing efficiencies $(\mathrm{P}=0.753$; Fig. 4G), erythrocyte-reducing efficiencies $(\mathrm{P}=0.998$; Fig. 4H) and basal CD62P expression rates of platelets $(\mathrm{P}=0.650$; Fig. 4I). However, the ADP-induced CD62P expression rate of platelets in P-PRP obtained by centrifugation at 250x15 was significantly higher than that obtained at $450 \times 10(\mathrm{P}=0.031)$ and 450x15 $(\mathrm{P}<0.001)$, but was comparable with that obtained at 180x10 $(\mathrm{P}>0.999), 180 \times 15(\mathrm{P}>0.999)$ and 250x10 $(\mathrm{P}>0.999$; Fig. 4J).

P-PRP obtained by centrifugation at $250 \times 15$ had a higher level of PDGF-AB compared to that obtained at $180 \times 10$ $(\mathrm{P}=0.001), 180 \times 15(\mathrm{P}=0.028), 250 \times 10(\mathrm{P}=0.008), 450 \times 10$ $(\mathrm{P}=0.011)$ and 450x15 ( $\mathrm{P}=0.004$; Fig. 5A). Similarly, P-PRP obtained at $250 \times 15$ had the highest level of TGF- $\beta 1$ compared 

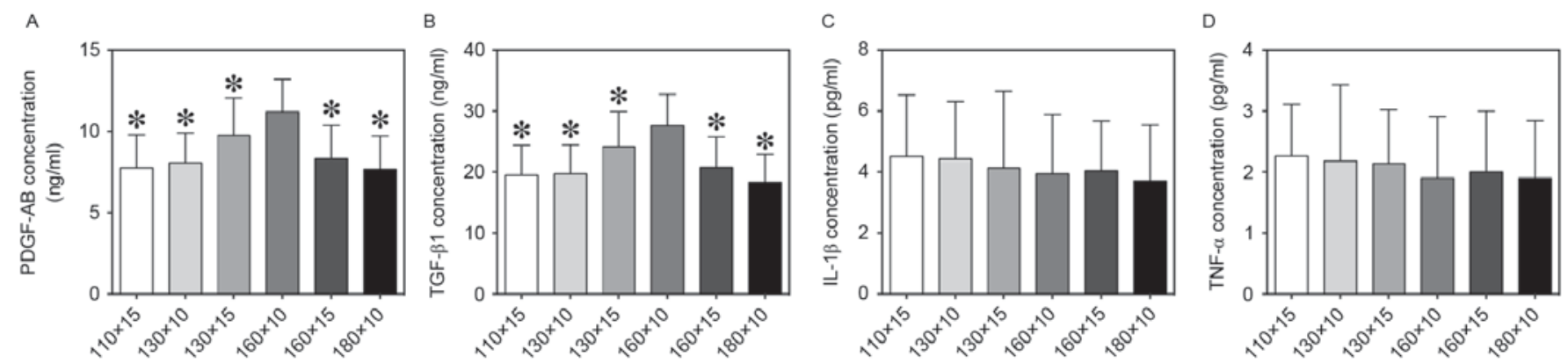

Figure 3. Cytokine concentrations in platelet-containing plasma obtained using different conditions. (A) PDGF-AB, (B) TGF- $\beta 1$, (C) IL-1 $\beta$ and (D) TNF- $\alpha$. Values are expressed as the mean \pm standard deviation. ${ }^{*} \mathrm{P}<0.05$ compared with $160 x 10$. PDGF-AB, platelet-derived growth factor; TGF- $\beta 1$, transforming growth factor $\beta 1$; IL-1 $\beta$, interleukin- $1 \beta$; TNF- $\alpha$, tumor necrosis factor- $\alpha$; $160 \times 10$, centrifugation at $160 \times \mathrm{x}$ for $10 \mathrm{~min}$.

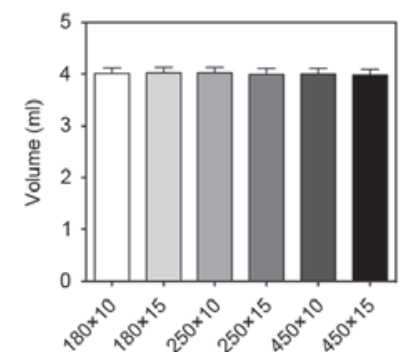

$E$
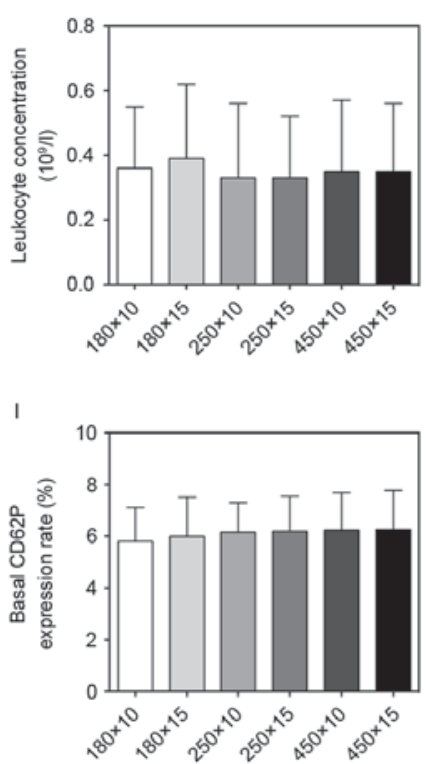
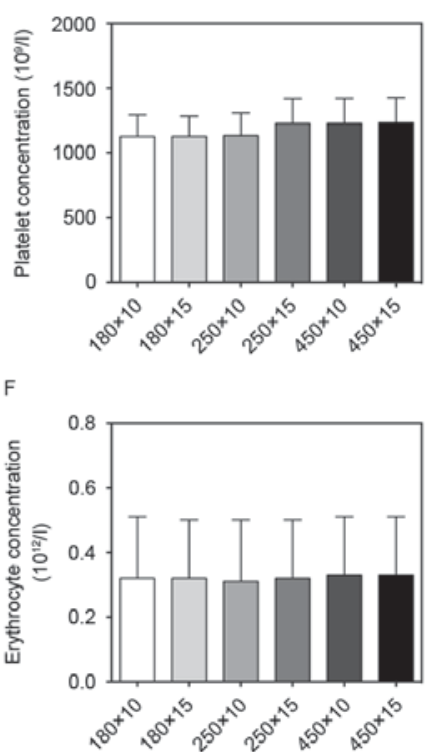

J

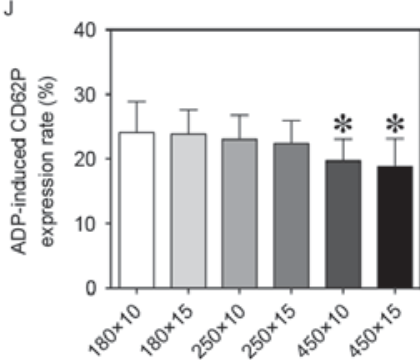

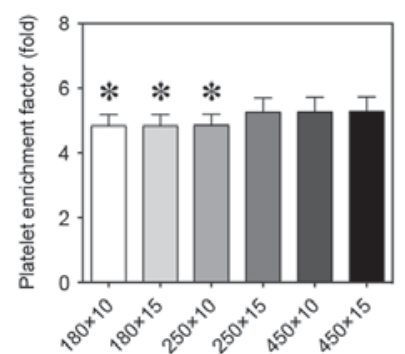

G

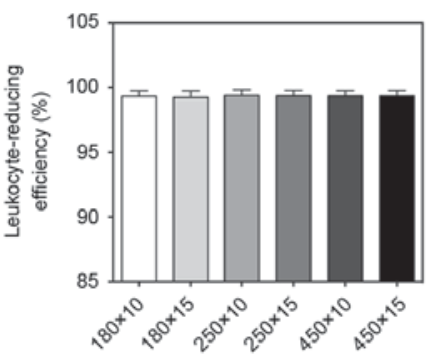

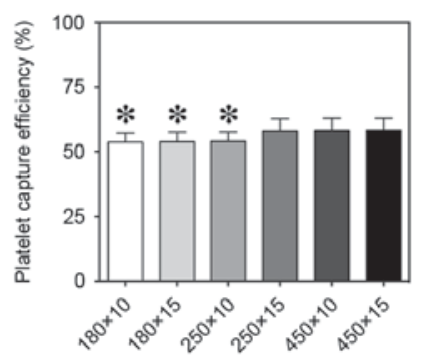

$\mathrm{H}$

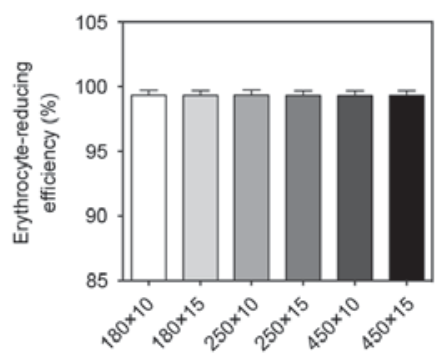

Figure 4. Characteristics of pure platelet-rich plasma obtained using different conditions. (A) Volume, (B) platelet concentration, (C) platelet enrichment factor, (D) platelet capture efficiency, (E) leukocyte concentration, (F) erythrocyte concentration, (G) leukocyte-reducing efficiency, (H) erythrocyte-reducing efficiency, (I) basal CD62P expression rate and (J) ADP-induced CD62P expression rate. Values are expressed as the mean \pm standard deviation. ${ }^{*} \mathrm{P}<0.05$ compared with 250x15. ADP, adenosine diphosphate; 250x15, centrifugation at $250 \mathrm{x} g$ for $15 \mathrm{~min}$.

with that obtained at $180 \times 10(\mathrm{P}=0.036), 180 \times 15(\mathrm{P}=0.007)$, 250x10 ( $\mathrm{P}=0.001), 450 \times 10(\mathrm{P}=0.002)$ and 450x15 $(\mathrm{P}<0.001$; Fig. 5B). Similar to the results regarding the leukocyte- and erythrocyte-associated characteristics of P-PRP, that obtained using different conditions had comparable concentrations of IL-1 $\beta$ ( $\mathrm{P}=0.695$; Fig. 5C) and TNF- $\alpha$ ( $\mathrm{P}=0.689$; Fig. 5D).

While providing comparable concentrations and reducing efficiencies of leukocytes and erythrocytes, pro-inflammatory cytokine concentrations and the basal platelet activation status to those obtained using other conditions, centrifugation at $250 \times 15$ enhanced the capture efficiency and enrichment factor of platelets of P-PRP to similar levels of those obtained using harder conditions (450x10 and 450x15) and diminished the harmful effects of centrifugation on the ADP-induced CD62P expression rates of platelets in P-PRP to similar levels of those achieved by softer conditions (180x10, 180x15 and 250x10). In addition, P-PRP obtained using 250x15 had the highest growth factor concentrations compared with that obtained using other 


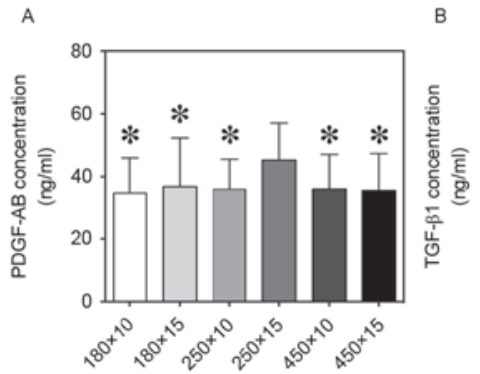

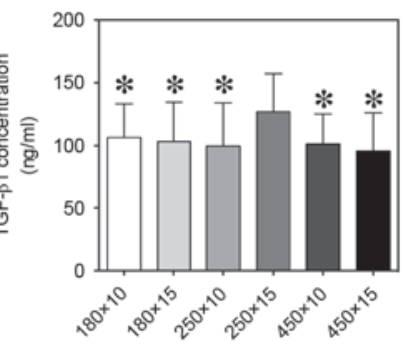

C

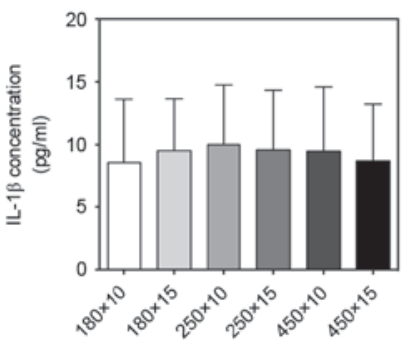

D

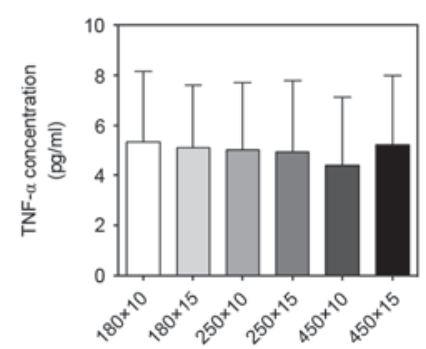

Figure 5. Cytokine concentrations of pure platelet-rich plasma obtained using different conditions. (A) PDGF-AB, (B) TGF- $\beta 1$, (C) IL-1 $\beta$ and (D) TNF- $\alpha$. Values are expressed as the mean \pm standard deviation. ${ }^{*} \mathrm{P}<0.05$ compared with $250 x 15$. ADP, adenosine diphosphate; PDGF-AB, platelet-derived growth factor; TGF- $\beta 1$, transforming growth factor $\beta 1$; IL-1 $\beta$, interleukin- $1 \beta$; TNF- $\alpha$, tumor necrosis factor- $\alpha$; $250 \times 15$, centrifugation at $250 \mathrm{x} g$ for $15 \mathrm{~min}$.

conditions. Therefore, the optimal centrifugation conditions for the second spin were determined to be $250 \times 15$.

Correlation between cytokine concentrations and the cellular characteristics of $P$-PRP. Significant positive correlations were observed between the PDGF-AB concentration in P-PRP and the platelet capture efficiency $(\mathrm{r}=0.133, \mathrm{P}=0.039$; Fig. $6 \mathrm{~A})$, platelet enrichment factor $(\mathrm{r}=0.159, \mathrm{P}=0.014$; Fig. $6 \mathrm{~B})$, the platelet concentration $(\mathrm{r}=0.532, \mathrm{P}<0.001$; Fig. $6 \mathrm{C})$ and the ADP-induced CD62P expression rate of platelets $(r=0.542$, $\mathrm{P}<0.001$; Fig. 6D).

Significant positive correlations were also observed between the TGF- $\beta 1$ concentration of P-PRP and the platelet capture efficiency $(\mathrm{r}=0.130, \mathrm{P}=0.044$; Fig. $6 \mathrm{E})$, platelet enrichment factor $(\mathrm{r}=0.158, \mathrm{P}=0.014$; Fig. $6 \mathrm{~F})$, platelet concentration $(r=0.493, P<0.001$; Fig. 6G) and the ADP-induced CD62P expression rate of platelets in $\mathrm{P}-\mathrm{PRP}(\mathrm{r}=0.555, \mathrm{P}<0.001$; Fig. 6H).

Furthermore, significant positive correlations were observed between the leukocyte concentration and the concentration of IL-1 $\beta(r=0.898, P<0.001$; Fig. 7A) and TNF- $\alpha$ in P-PRP $(r=0.863, \mathrm{P}<0.001$; Fig. 7B). Significant negative correlations were observed between the leukocyte-reducing efficiency of P-PRP and the concentration of IL-1 $\beta$ ( $r=-0.803$, $\mathrm{P}<0.001$; Fig. 7C) and TNF- $\alpha$ in P-PRP ( $\mathrm{r}=-0.774, \mathrm{P}<0.001$; Fig. 7D).

Effects of P-PRP obtained using different conditions on cells in vitro. The proliferation of hBMSCs in the presence of P-PRP obtained using different conditions was comparable on day 1 ( $\mathrm{P}=0.724$; Fig. 8A). However, compared with P-PRP obtained using other conditions, that obtained by second-spin centrifugation at $250 \times 15$ significantly promoted the proliferation of hBMSCs after incubation for 4 days $(\mathrm{P}=0.001)$ and 7 days $(\mathrm{P}<0.001)$. Similar results were also observed regarding the proliferation of hACs in the presence of P-PRP, which was comparable between groups on day $1(\mathrm{P}=0.805$; Fig. $8 \mathrm{~B})$ and significantly enhanced by P-PRP obtained by second-spin centrifugation at $250 \times 15$ on day $4(\mathrm{P}=0.028)$ and day 7 $(\mathrm{P}=0.005)$.

The live/dead staining results for hBMSCs and hACs are shown in Fig. 8C. Quantified analysis revealed that P-PRP obtained using 250x15 maintained the survival of hBMSCs and hACs to a comparable extent to P-PRP obtained using other conditions $(\mathrm{P}=0.384$ and $\mathrm{P}=0.627$, respectively; Fig. $8 \mathrm{D})$.
Representative images of migrated hBMSCs and hACs are shown in Fig. 9A. Quantified analysis revealed that P-PRP obtained by $250 \times 15$ significantly promoted the migration of hBMSCs $(\mathrm{P}<0.001)$ and hACs $(\mathrm{P}<0.001$; Fig. 9B) compared with P-PRP obtained obtained using other conditions.

\section{Discussion}

The study by Bausset et al (10) demonstrated that centrifugation at 250x15 min, which is not used by any of the currently existing preparation systems, maximizes platelet enrichment while preserving the bioactivity of platelets. However, this method did not achieve the same platelet enrichment as that in leukocyte- and platelet-rich plasma, which concentrates platelets and leukocytes 4- to 8-fold that of the baseline levels (6). The possible reason may be that the centrifugal conditions of the first spin of the system used by Bausset et al (10), namely $130 \times 15$, may not be the optimal conditions to capture platelets (15). The results of the present study demonstrated that the first spin at 160x10 captured more platelets than that at 130x15. Therefore, although the optimal centrifugal condition for the second spin determined by the present study $(250 \times 15)$ were in accordance with those of the study by Bausset et al (10), the higher platelet capture efficiency of the first spin captured more platelets to be concentrated with the second spin, resulting in a P-PRP with a higher platelet concentration and enrichment factor. Therefore, 160x10 may be more appropriate for the first spin than $130 \times 15$.

It is well known that the buffy coat contains concentrated leukocytes and complete removal of the buffy coat is necessary to deplete leukocytes from the final PRP obtained (19). However, the interface of buffy coat and PCP is not clear and PCP is generally contaminated by a portion of buffy coat. Due to the fact that the buffy coat also contains concentrated platelets, contamination with the buffy coat may result in a higher platelet capture efficiency of the final P-PRP obtained and therefore result in a higher platelet concentration and enrichment factor of the P-PRP obtained. Thus, the completeness of buffy coat removal is a potential confounding variable for the comparison of the effects of centrifugal conditions on the cellular composition of P-PRP obtained. In the present study, the same operator performed all of the procedures for buffy coat removal to avoid artifacts due to the difference of completeness of buffy coat removal. The results demonstrated that PCP and P-PRP obtained using different conditions 

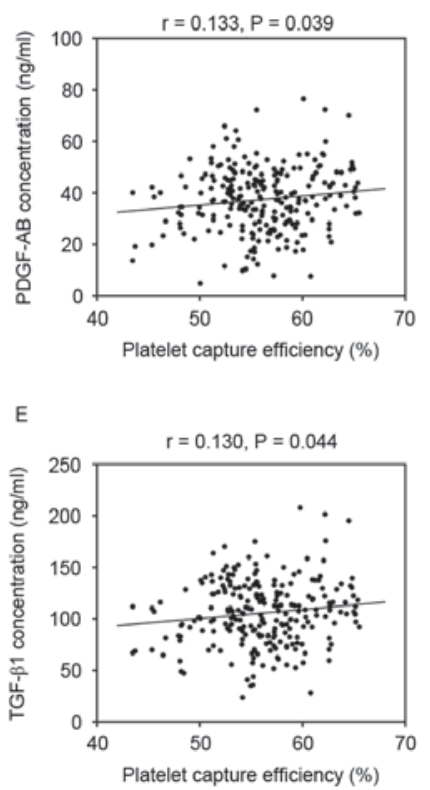
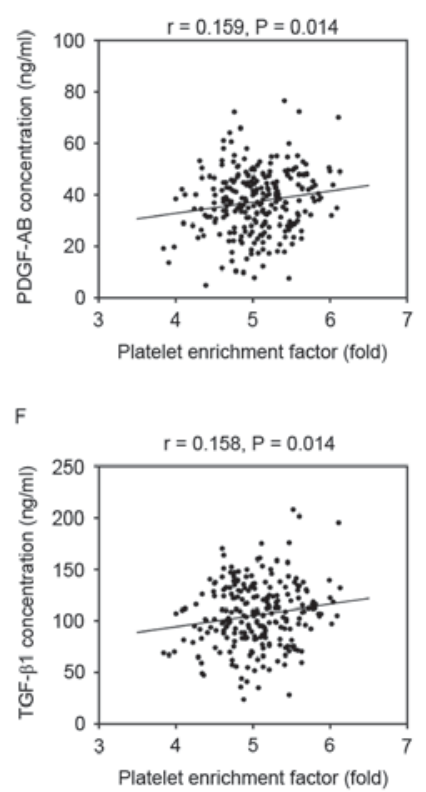

C

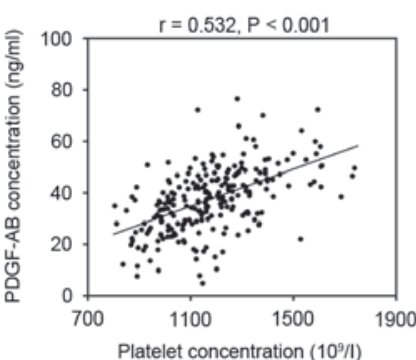

G

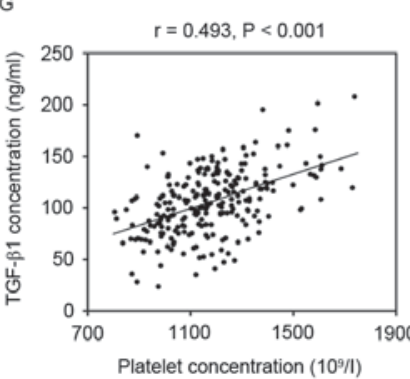

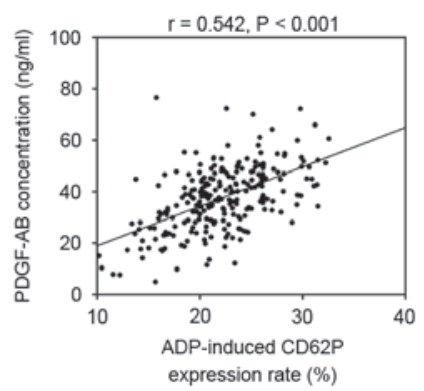

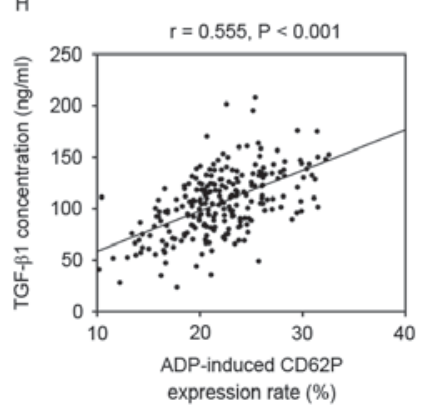

Figure 6. Correlation between growth factor concentrations and the characteristics of P-PRP. Positive correlations between the PDGF-AB concentration in P-PRP and (A) the platelet concentration, (B) platelet enrichment factor, (C) platelet capture efficiency and (D) the ADP-induced CD62P expression rate in P-PRP were detected. Furthermore, positive correlations between the TGF- $\beta 1$ concentration in P-PRP and (E) the platelet concentration, (F) platelet enrichment factor, $(\mathrm{G})$ platelet capture efficiency and $(\mathrm{H})$ the ADP-induced CD62P expression rate in P-PRP were detected. PDGF-AB, platelet-derived growth factor; TGF- $\beta 1$, transforming growth factor $\beta 1$; P-PRP, pure platelet-rich plasma; ADP, adenosine diphosphate.

A

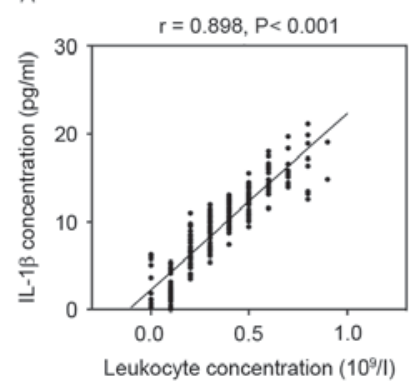

B

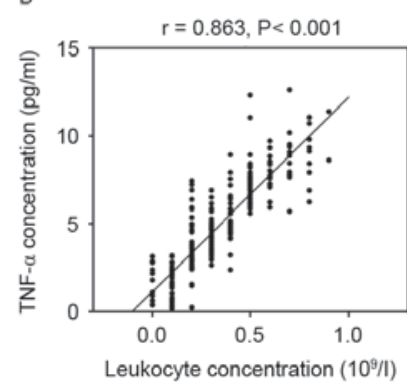

C

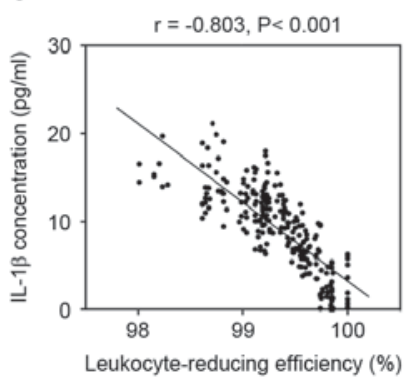

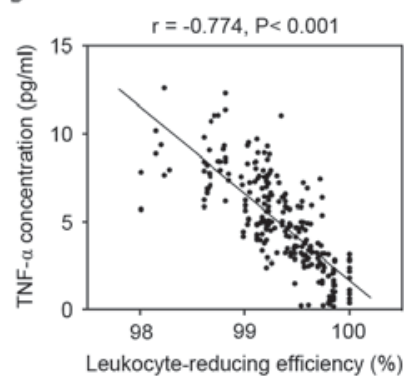

Figure 7. Correlations between pro-inflammatory cytokine concentrations and the characteristics of P-PRP. Positive correlations between the leukocyte concentration in P-PRP and (A) the IL-1 $\beta$ concentration and (B) the TNF- $\alpha$ concentration in P-PRP were identified. Negative correlations between the leukocyte-reducing efficiency of P-PRP and (C) the IL-1 $\beta$ concentration and (D) the TNF- $\alpha$ concentration in P-PRP were detected. IL-1 $\beta$, interleukin- $1 \beta$; TNF- $\alpha$, tumor necrosis factor- $\alpha$; P-PRP, pure platelet-rich plasma.

had similar leukocyte and erythrocyte concentrations. The results revealed that the completeness of buffy coat removal was constant in the process of the experiments. Thus, it is plausible to conclude that the different platelet-associated characteristics of P-PRP from different centrifugal conditions resulted from the difference of centrifugal conditions, rather than the difference in the completeness of buffy coat removal.

Both PDGF-AB and TGF- $\beta 1$ have been shown to be desirable in tissue healing $(1,13,20-22)$. Therefore, the present study assessed PDGF-AB and TGF- $\beta 1$ concentrations in PCP and P-PRP obtained using different conditions. The results demonstrated that the first spin at $160 \times 10$, which captured more platelets than the other conditions for the first spin, further concentrated growth factors. Different from the results of the first spin, even though the second spin at 250x15 neither captured more platelets nor concentrated platelets further than harder conditions for the second spin $(450 \times 10$ and 450x15), it achieved a further concentration of growth factors. The possible reason for this phenomenon may be that the concentrations of growth factors are associated not only with the concentrations, enrichment factors and capture efficiencies of platelets $(1,8)$, but also with the exogenous activation-induced CD62P expression rates (23), as demonstrated by previous studies as well as the correlation analysis of the present study. Hence, the lower ADP-induced CD62P expression rates of platelets in P-PRP obtained by using

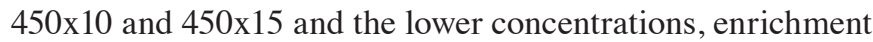
factors and capture efficiencies of platelets in P-PRP obtained using 180x10, 180x15 and 250x15 may account for the lower concentrations of growth factors. Therefore, the preservation of the platelet reactivity to exogenous activation may have 
A
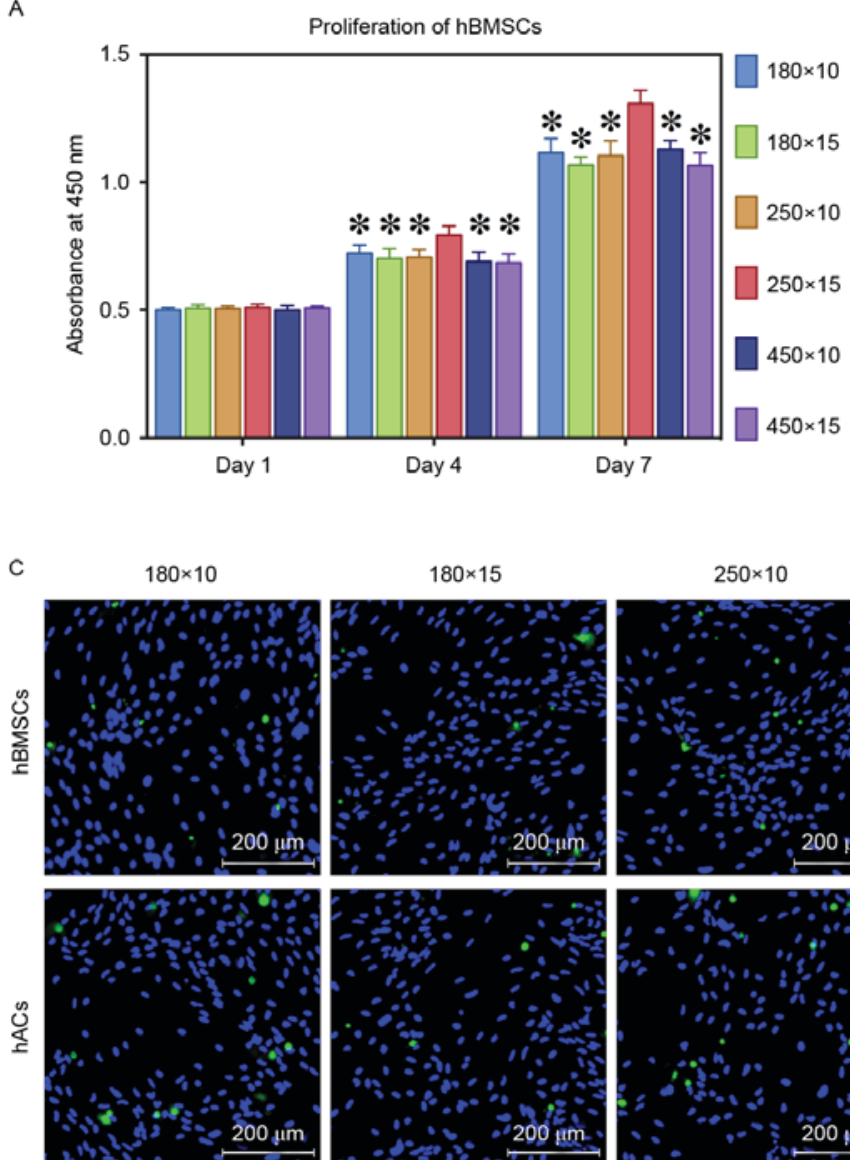

$180 \times 15$
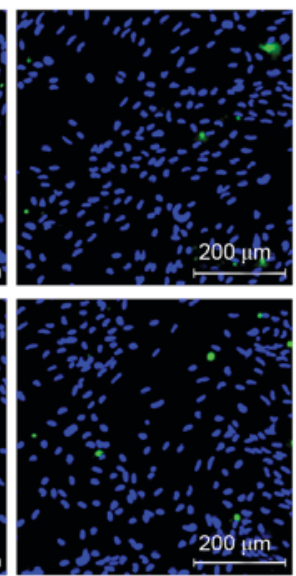

$250 \times 10$
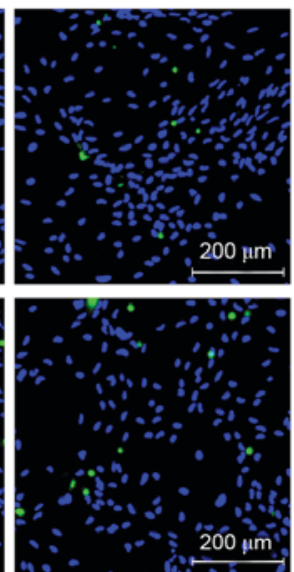

B

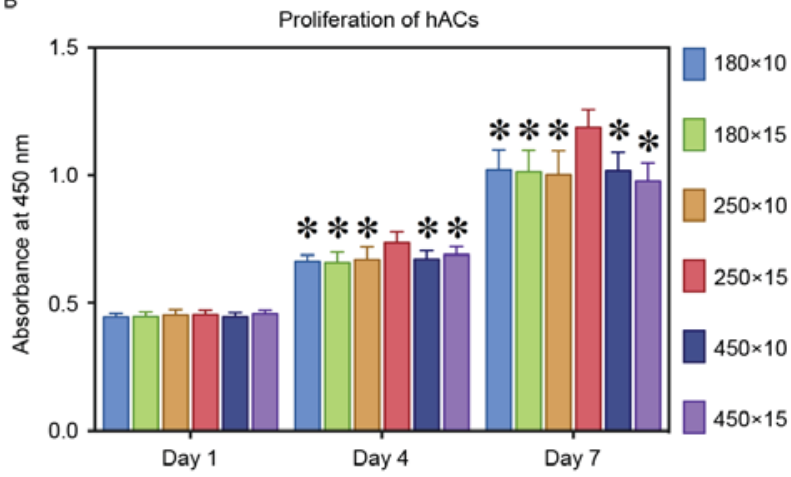

$250 \times 15$

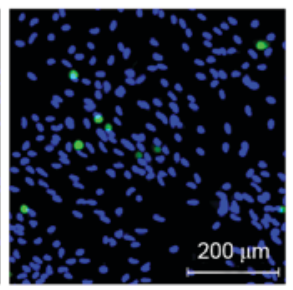

$450 \times 10$
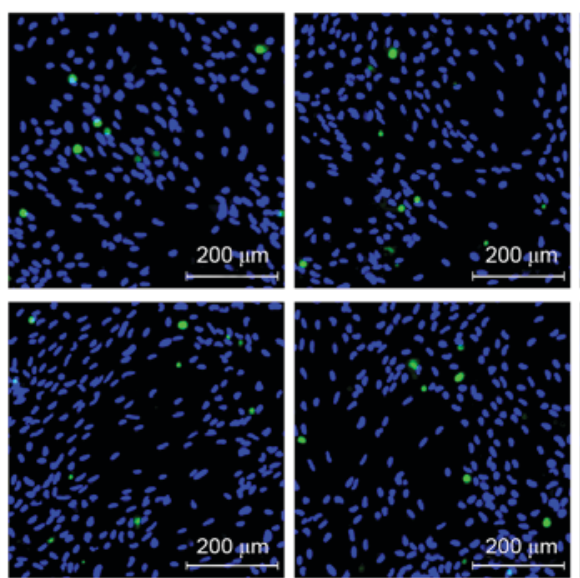

$450 \times 15$

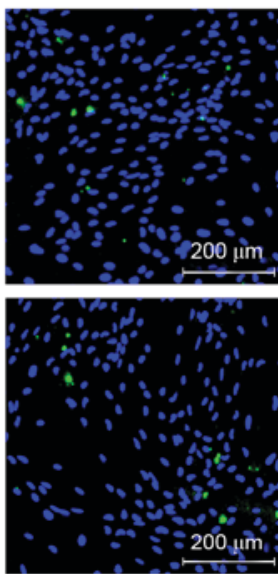

D

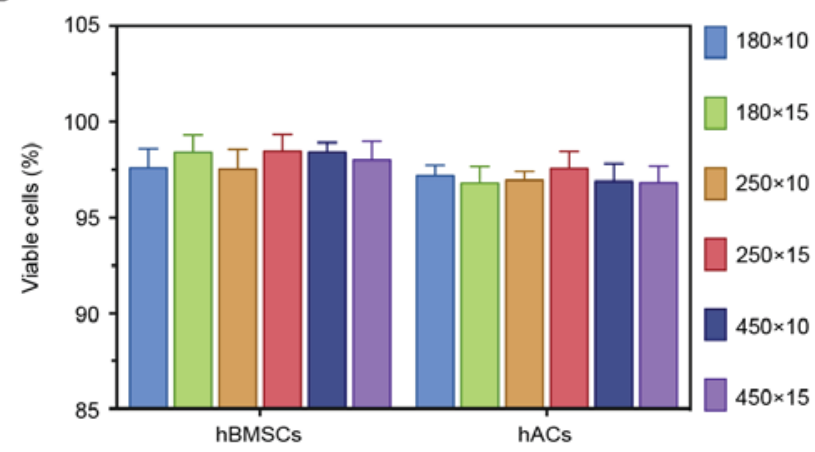

Figure 8. Effects of P-PRP on the proliferation and survival of hBMSCs and hACs. A Cell Counting Kit- 8 assay demonstrated that P-PRP obtained by $250 x 15$ centrifugation significantly promoted the proliferation of (A) hBMSCs and (B) hACs after incubation for 4 and 7 days compared with P-PRP obtained using other conditions. (C) Representative images of hBMSCs and hACs subjected to cell viability imaging assay. Viable cells stained blue and dead cells stained green (scale bar, $200 \mu \mathrm{m}$ ). (D) Quantitative analysis of live/dead staining revealed that P-PRP obtained using different conditions had similar effects on the survival of hBMSCs and hACs. Values are expressed as the mean \pm standard deviation. " $\mathrm{P}<0.05$ compared with $250 \mathrm{x} 15$. hBMSCs, human bone marrow-derived mesenchymal stem cells; hACs, human articular chondrocytes; P-PRP, pure platelet-rich plasma; 250x15, second-spin centrifugation at $250 \mathrm{x} g$ for 15 min.

an equally important role as the enrichment and capture of platelets in P-PRP preparation (24).

IL-1 $\beta$ and TNF- $\alpha$ are the primary cytokines for inflammation and matrix degradation $(8,13,20,25)$. Therefore, the present study also assessed the concentration of IL-1 $\beta$ and TNF- $\alpha$ in PCP and P-PRP obtained using different conditions. The results demonstrated that centrifugation at $160 \times 10$ and 250x15 achieved leukocyte concentrations, leukocyte-reducing efficiencies, IL-1 $\beta$ concentration and TNF- $\alpha$ concentration in P-PRP similar to those obtained using other conditions. The results of the correlation analysis were in accordance with those of previous studies demonstrating that the leukocyte reduction resulted in decreased concentrations of pro-inflammatory cytokines $(1,8)$. The results implied that buffy coat removal employed by the present study was the crucial factor for the reduction of pro-inflammatory cytokines and that the centrifugal conditions may not have influenced the reduction of leukocytes and pro-inflammatory cytokines as long as the buffy coat was discarded. Therefore, centrifugation at 160x10 and 250x15 with removal of the buffy coat as a crucial step may theoretically be the optimal method for preparing P-PRP. 

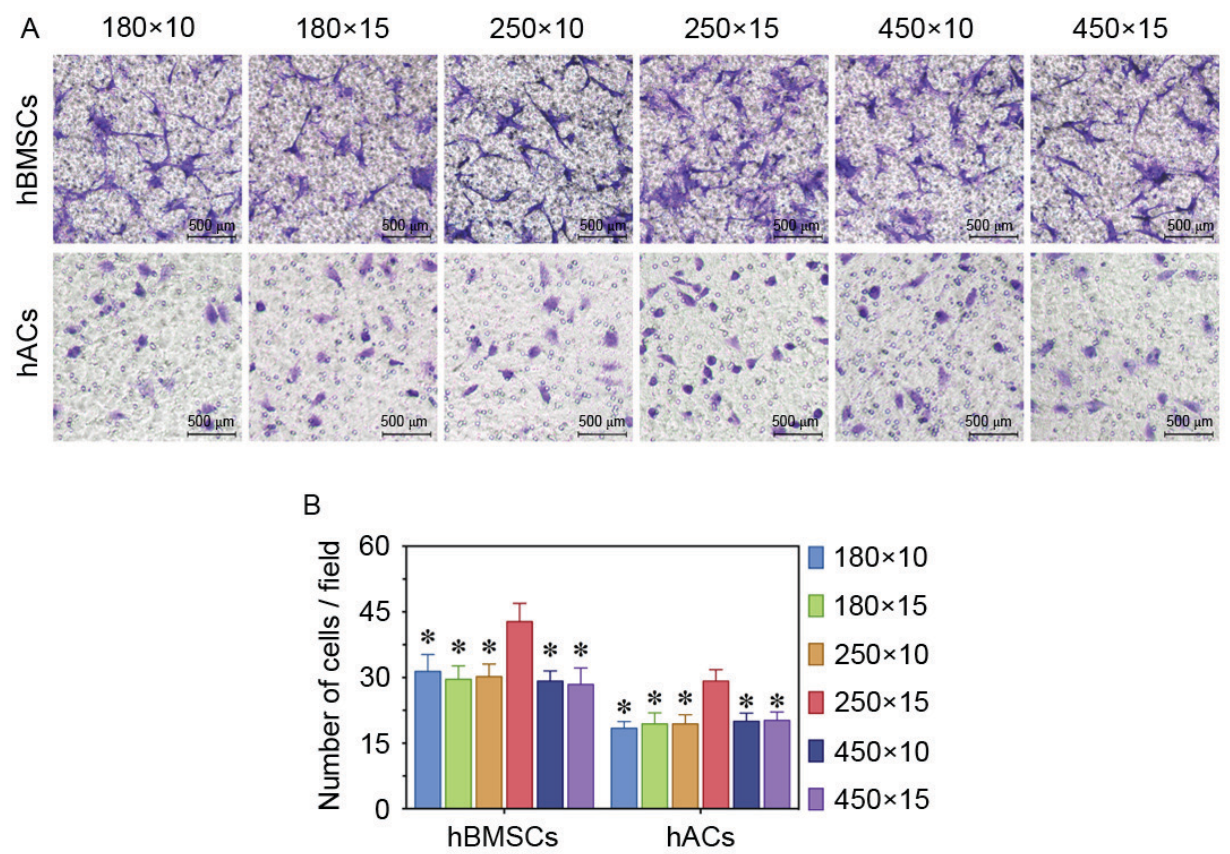

Figure 9. Pure platelet-rich plasma obtained by 250x15 promoted the migration of hBMSCs and hACs. (A) Migrated hBMSCs and hACs were stained with crystal violet (scale bar, $500 \mu \mathrm{m}$ ); (B) the migration of hBMSCs and hACs was expressed as the number of migrated cells per field. Values are expressed as the mean \pm standard deviation. ${ }^{*} \mathrm{P}<0.05$ compared with $250 \times 15.250 \times 15$, second-spin centrifugation at $250 \mathrm{x} g$ for 15 min; hBMSCs, human bone marrow-derived mesenchymal stem cells; hACs, human articular chondrocytes.

However, the optimal P-PRP for tissue healing is not born with the optimal P-PRP preparation system. There is no consensus on the optimal platelet concentration of PRP for tissue healing and certain authors suggested that the optimal platelet concentration and enrichment for healing of each tissue may be different (13). PRP has been widely used in the treatment of bone defects $(26,27)$, osteochondral defects $(28,29)$ and arthritis $(30,31)$. Therefore, hBMSCs and hACs were selected to evaluate the effects of P-PRP in vitro. The results demonstrated that P-PRP obtained using the optimal conditions significantly promoted the proliferation and migration of hBMSCs and hACs compared with P-PRP obtained using other conditions. Numerous studies have demonstrated that the promotion of proliferation and migration of hBMSCs is beneficial for the regeneration of bone (25) and cartilage (32) and that the promotion of proliferation and migration of hACs is beneficial for the treatment of arthritis (33). Therefore, P-PRP obtained using the optimal conditions identified may also be more effective in the treatment of bone defects, osteochondral defects and arthritis. However, further studies are required to substantiate this in vivo and in vitro.

In conclusion, while P-PRP obtained using different centrifugal conditions had similar erythrocyte, leukocyte and pro-inflammatory cytokine concentrations, centrifugation at 160x10 and 250x15 successively captured and concentrated platelets and growth factors more efficiently with preservation of platelet function compared with other conditions. Moreover, P-PRP obtained using the optimal conditions significantly promoted the proliferation and migration of cells and did not alter cell survival compared with P-PRP obtained from other conditions. Therefore, centrifugation at $160 \times 10$ and 250x15 successively with removal of the buffy coat as a crucial step may be able to provide an optimal method for the preparation of P-PRP for clinical application.

\section{Acknowledgements}

The present study was supported by the National Natural Science Foundation of China (grant no. 81401799) and the Shanghai Youth Start-up Grant (grant no. 14YF1412100).

\section{References}

1. Castillo TN, Pouliot MA, Kim HJ and Dragoo JL: Comparison of growth factor and platelet concentration from commercial platelet-rich plasma separation systems. Am J Sports Med 39: 266-271, 2011.

2. Xie X, Zhang C and Tuan RS: Biology of platelet-rich plasma and its clinical application in cartilage repair. Arthritis Res Ther 16: 204, 2014.

3. Martin JI, Merino J, Atilano L, Areizaga LM, Gomez-Fernandez MC, Burgos-Alonso N and Andia I: Platelet-rich plasma (PRP) in chronic epicondylitis: Study protocol for a randomized controlled trial. Trials 14: 410, 2013.

4. Guelfi M, Pantalone A, Vanni D, Abate M, Guelfi MG and Salini V: Long-term beneficial effects of platelet-rich plasma for non-insertional Achilles tendinopathy. Foot Ankle Surg 21: 178-181, 2015.

5. Intini G, Andreana S, Intini FE, Buhite RJ and Bobek LA: Calcium sulfate and platelet-rich plasma make a novel osteoinductive biomaterial for bone regeneration. J Transl Med 5: 13, 2007.

6. Filardo G, Kon E, Pereira Ruiz MT, Vaccaro F, Guitaldi R, Di Martino A, Cenacchi A, Fornasari PM and Marcacci M: Platelet-rich plasma intra-articular injections for cartilage degeneration and osteoarthritis: Single-versus double-spinning approach. Knee Surg Sports Traumatol Arthrosc 20: 2082-2091, 2012.

7. McCarrel TM, Minas T and Fortier LA: Optimization of leukocyte concentration in platelet-rich plasma for the treatment of tendinopathy. J Bone Joint Surg Am 94: e143, 2012.

8. Sundman EA, Cole BJ and Fortier LA: Growth factor and catabolic cytokine concentrations are influenced by the cellular composition of platelet-rich plasma. Am J Sports Med 39: 2135-2140, 2011. 
9. Jo CH, Shin JS, Lee YG, Shin WH, Kim H, Lee SY, Yoon KS and Shin S: Platelet-rich plasma for arthroscopic repair of large to massive rotator cuff tears: A randomized, single-blind, parallel-group trial. Am J Sports Med 41: 2240-2248, 2013.

10. Bausset O, Giraudo L, Veran J, Magalon J, Coudreuse JM, Magalon G, Dubois C, Serratrice N, Dignat-George F and Sabatier F: Formulation and storage of platelet-rich plasma homemade product. Biores Open Access 1: 115-123, 2012.

11. Sanchez M, Yoshioka T, Ortega M, Delgado D and Anitua E: Ultrasound-guided platelet-rich plasma injections for the treatment of common peroneal nerve palsy associated with multiple ligament injuries of the knee. Knee Surg Sports Traumatol Arthrosc 22: 1084-1089, 2014.

12. Mazzocca AD, McCarthy MB, Chowaniec DM, Cote MP, Romeo AA, Bradley JP, Arciero RA and Beitzel K: Platelet-rich plasma differs according to preparation method and human variability. J Bone Joint Surg Am 94: 308-316, 2012.

13. Arnoczky SP and Sheibani-Rad S: The basic science of platelet-rich plasma (PRP): What clinicians need to know. Sports Med Arthrosc 21: 180-185, 2013.

14. Stokes GG: On some cases of fluid motion. Trans Camb Philos Soc 8: 105-165, 1843.

15. Magalon J, Bausset O, Serratrice N, Giraudo L, Aboudou H, Veran J, Magalon G, Dignat-Georges F and Sabatier F: Characterization and comparison of 5 platelet-rich plasma preparations in a single-donor model. Arthroscopy 30: 629-638, 2014.

16. Soleimani M and Nadri S: A protocol for isolation and culture of mesenchymal stem cells from mouse bone marrow. Nat Protoc 4 102-106, 2009

17. Zhu H, Cheng X, Niu X, Zhang Y, Guan J, Liu X, Tao S, Wang Y and Zhang C: Proton-sensing GPCR-YAP Signalling Promotes Cell Proliferation and Survival. Int J Biol Sci 11: 1181-1189, 2015.

18. Liu YS, Ou ME, Liu H, Gu M, Lv LW, Fan C, Chen T, Zhao XH, Jin CY, Zhang X, et al: The effect of simvastatin on chemotactic capability of SDF- $1 \alpha$ and the promotion of bone regeneration. Biomaterials 35: 4489-4498, 2014.

19. Arnoczky SP, Delos D and Rodeo SA: What is platelet-rich plasma? Oper Tech Sports Med 19: 142-148, 2011

20. Andia I and Abate M: Platelet-rich plasma: Underlying biology and clinical correlates. Regen Med 8: 645-658, 2013.

21. Browning SR, Weiser AM, Woolf N, Golish SR, SanGiovanni TP, Scuderi GJ, Carballo C and Hanna LS: Platelet-rich plasma increases matrix metalloproteinases in cultures of human synovial fibroblasts. J Bone Joint Surg Am 94: e1721-e1727, 2012.

22. Cavallo C, Filardo G, Mariani E, Kon E, Marcacci M, Pereira Ruiz MT, Facchini A and Grigolo B: Comparison of platelet-rich plasma formulations for cartilage healing: An in vitro study. J Bone Joint Surg Am 96: 423-429, 2014
23. Graff J, Klinkhardt U, Schini-Kerth VB, Harder S, Franz N, Bassus $S$ and Kirchmaier CM: Close relationship between the platelet activation marker CD62 and the granular release of platelet-derived growth factor. J Pharmacol Exp Ther 300: 952-957, 2002.

24. Dohan Ehrenfest DM, Rasmusson L and Albrektsson T: Classification of platelet concentrates: From pure platelet-rich plasma (P-PRP) to leucocyte- and platelet-rich fibrin (L-PRF). Trends Biotechnol 27: 158-167, 2009.

25. Andersen RK, Zaher W, Larsen KH, Ditzel N, Drews K, Wruck W, Adjaye J, Abdallah BM and Kassem M: Association between in vivo bone formation and ex vivo migratory capacity of human bone marrow stromal cells. Stem Cell Res Ther 6: 196, 2015.

26. Lee DH, Ryu KJ, Kim JW, Kang KC and Choi YR: Bone marrow aspirate concentrate and platelet-rich plasma enhanced bone healing in distraction osteogenesis of the tibia. Clin Orthop Relat Res 472: 3789-3797, 2014.

27. Kim YH, Furuya $\mathrm{H}$ and Tabata Y: Enhancement of bone regeneration by dual release of a macrophage recruitment agent and platelet-rich plasma from gelatin hydrogels. Biomaterials 35 : 214-224, 2014.

28. Milano G, Sanna Passino E, Deriu L, Careddu G, Manunta L, Manunta A, Saccomanno MF and Fabbriciani C: The effect of platelet rich plasma combined with microfractures on the treatment of chondral defects: An experimental study in a sheep model. Osteoarthritis Cartilage 18: 971-980, 2010.

29. Mei-Dan O, Carmont MR, Laver L, Mann G, Maffulli N and Nyska M: Platelet-rich plasma or hyaluronate in the management of osteochondral lesions of the talus. Am J Sports Med 40: 534-541, 2012.

30. Patel S, Dhillon MS, Aggarwal S, Marwaha N and Jain A: Treatment with platelet-rich plasma is more effective than placebo for knee osteoarthritis: A prospective, double-blind, randomized trial. Am J Sports Med 41: 356-364, 2013.

31. Kon E, Mandelbaum B, Buda R, Filardo G, Delcogliano M, Timoncini A, Fornasari PM, Giannini S and Marcacci M: Platelet-rich plasma intra-articular injection versus hyaluronic acid viscosupplementation as treatments for cartilage pathology: From early degeneration to osteoarthritis. Arthroscopy 27: 1490-1501, 2011.

32. Liu J, Nie H, Xu Z, Guo F, Guo S, Yin J, Wang Y and Zhang C: Construction of PRP-containing nanofibrous scaffolds for controlled release and their application to cartilage regeneration. J Materials Chem B 3: 581-591, 2015.

33. Pereira RC, Scaranari M, Benelli R, Strada P, Reis RL, Cancedda R and Gentili C: Dual effect of platelet lysate on human articular cartilage: A maintenance of chondrogenic potential and a transient proinflammatory activity followed by an inflammation resolution. Tissue Eng Part A 19: 1476-1488, 2013 